\title{
Comunicación
}

\section{Cólico Equino por Impacción Gástrica en una Yegua Pura Sangre Inglés}

\author{
Equine Colic Due to Gastric Impaction in a Thoroughbred Mare
}

Katherine Choez A. ${ }^{1,2}$, Rocío Sandoval M. ${ }^{1}$, Luis Ruiz G. ${ }^{1}$, Alfredo Delgado C. ${ }^{1}$

\section{Resumen}

El presente reporte describe un caso clínico de cólico que terminó en muerte, por impacción gástrica de origen alimenticio ocurrido una yegua de 12 años. Este caso se desencadenó por el consumo accidental y voraz de alimento concentrado para alpacas durante un descuido del personal. El cuadro clínico se caracterizó por depresión severa, dolor abdominal intenso, decúbito lateral e incapacidad para levantarse, siendo el curso rápido, de pronóstico grave, que devino en muerte. Las lesiones macroscópicas incluyeron distensión severa del estómago, el cual estaba impactado con abundante contenido parcialmente digerido, fibroso y seco, con úlceras en la mucosa y presencia de exudado seroso en la cavidad abdominal.

Palabras clave: cólico, impacción, gástrica, equino

\section{Abstract}

This paper describes a case of colic that ended in death due to gastric impaction occurred to a mare of 12 years of age. This case was triggered by the accidental and voracious consumption of concentrate feed intended for alpacas during a failure of supervision by staff. The clinical picture was characterized by severe depression, severe abdominal pain, lateral decubitus and inability to rise, with a rapid course and poor prognosis which turned fatal. Gross lesions included severe stomach distension, which was impacted with plenty content partially digested, fibrous and dry, with mucosal ulcerations and presence of serous exudate in the abdominal cavity.

Key words: colic, impaction, gastric, equine

${ }^{1}$ Clínica de Animales Mayores, Facultad de Medicina Veterinaria, Universidad Nacional Mayor de San Marcos, Lima, Perú

2E-mail: kathyvet3@hotmail.com

Recibido: 8 de agosto de 2016

Aceptado para publicación: 15 de noviembre de 2016 


\section{INTRODUCCIÓN}

El cólico, más conocido como dolor abdominal agudo, es un término inespecífico dada la gran variedad de causas (abdominales y no abdominales) que pueden llevar a la presencia de los signos clínicos (Ferreira et al., 2007). En la clínica veterinaria equina, la impacción gástrica debida a un gran acúmulo de forraje fibroso de baja digestibilidad o concentrado altamente fermentable es una de las causas más comunes de cólico (White, 2005).

Los signos clínicos pueden aparecer de repente o bien ser sutiles e ir progresando a lo largo del día. Estos signos varían, dependiendo de la localización, gravedad y causa del dolor. Frecuentemente, se observa depresión e inapetencia; asimismo, el animal suele mirarse los flancos, tumbarse y levantarse repetidas veces o revolcarse, rascar en el suelo y pegarse patadas al abdomen, y en casos graves, adopta una posición parecida a la de un perro sentado. Además, hay disminución de la producción de heces o heces secas y duras, sudoración excesiva y aumento de la frecuencia respiratoria y cardiaca (Smith, 2010).

El éxito en el manejo del cólico se basa en su rápido diagnóstico y tratamiento efectivo, que puede ser médico o quirúrgico. Sin embargo, cuando se exceden los periodos de tiempo normales de resolución del mismo, el problema se torna más grave y puede sobrevenir la muerte.

\section{Caso Clínico}

En mayo de 2015, una yegua pura sangre inglés, de 12 años de edad y de aproximadamente $350 \mathrm{~kg}$ de peso vivo fue observada con signos evidentes de cólico. Según los datos de la anamnesis realizada, su alimentación era a base de heno de alfalfa y agua a voluntad.
En horas de la noche, el animal presentaba anorexia y signos de dolor agudo tirándose de forma brusca al suelo; además, no había señales de que hubiera defecado. A la mañana siguiente, la yegua se encontraba muy deprimida, estaba en decúbito lateral y era incapaz de levantarse. El examen clínico reveló mucosas oculares congestionadas y secas, tiempo de llenado capilar de la mucosa gingival mayor a 4 segundos, frecuencia cardiaca y de pulso de $105 \mathrm{lpm}$ y $98 \mathrm{ppm}$, respectivamente, frecuencia respiratoria de 80 rpm y de tipo costal, deshidratación evidente con recuperación de la elasticidad de la piel de la tabla del cuello en 5 segundos y temperatura rectal de $37^{\circ} \mathrm{C}$. Además, el abdomen se encontraba distendido y sin sonidos audibles a la auscultación de los flancos.

El personal encargado de la alimentación le administró cloruro de sodio al $0.9 \%$ y flunixin meglumine $(1.1 \mathrm{mg} / \mathrm{kg})$ vía endovenosa, sin que hubiese signos de mejoría, posiblemente debido a la demora en el inicio del tratamiento. El animal tenía un pronóstico desfavorable, por lo que para evitar su sufrimiento se practicó la eutanasia con pentobarbital sódico al 20\% (40-60 mg/kg), vía endovenosa, y sulfato de magnesio diluido, vía intracardiaca.

En la necropsia, realizada a una hora del deceso, se observaron múltiples hematomas localizados en el párpado superior derecho, a nivel de la articulación húmeroradio-ulnar derecha y alrededor de la $6^{\mathrm{a}}$ y $7^{\mathrm{a}}$ costilla del hemitórax derecho, con fractura completa de estas. Además, se observó el estómago severamente distendido, repleto de contenido fibroso y seco parcialmente digerido, con pequeñas lesiones ulcerosas en la mucosa gástrica, así como una gastritis catarro-hemorrágica.

El intestino se encontraba distendido con contenido líquido amarillento y edema generalizado. Asimismo, había presencia de exudado seroso en la cavidad abdominal. El 


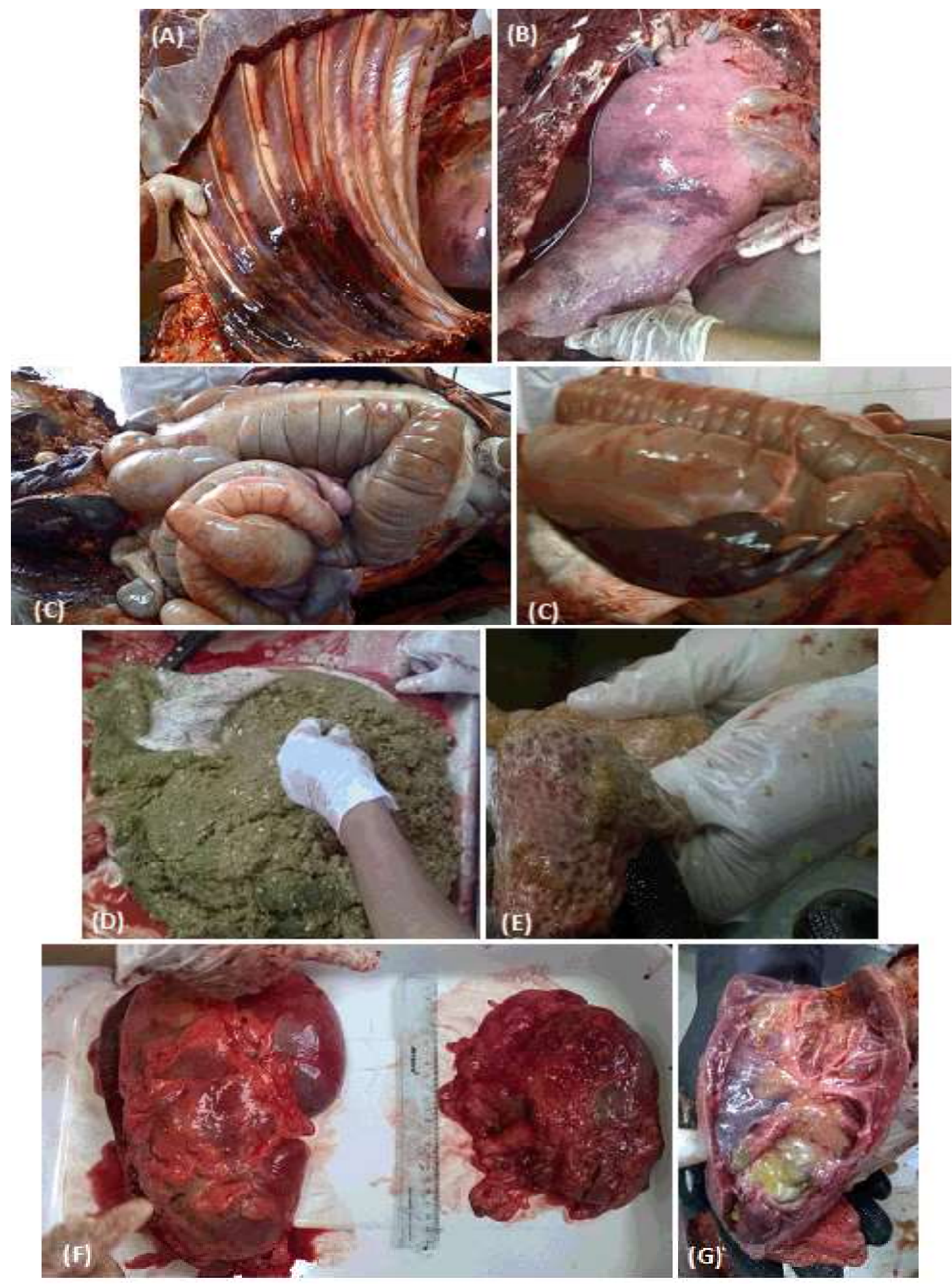

Figura 1.Hallazgos macroscópicos en la necropsia. A) Hematoma y fractura a nivel de la $6^{\mathrm{a}}$ y $7^{\mathrm{a}}$ costilla derecha; B) Áreas congestionadas en pulmón; C) Distensión de vísceras abdominales; D) Estómago repleto de contenido fibroso y seco parcialmente digerido; E) Úlcera en mucosa gástrica; F) Riñón derecho más pequeño que el izquierdo; G) Corte del riñón derecho con presencia de urolito y contenido purulento 
riñón izquierdo era de tamaño y aspecto normal; sin embargo, el riñón derecho era más pequeño que el izquierdo y presentaba múltiples abscesos focales y al corte se observaron urolitos y contenido purulento. El corazón e hígado tenían un aspecto normal y los pulmones presentaban áreas de congestión.

Tras la investigación realizada, se determinó que también se criaban alpacas en el lugar donde vivía la yegua, y que el almacén del alimento para las alpacas se encontraba cerca de los boxes de los caballos. El día en que ocurrió el problema, el personal encargado de la alimentación observó que la yegua se encontraba fuera de su box y horas más tarde, este mismo personal, también se percató que la puerta del almacén estaba abierta y que uno de los sacos del alimento concentrado para alpacas estaba roto. Es de esa forma que se pudo establecer que la yegua tuvo acceso accidental al alimento concentrado, por lo que la ingestión en exceso ocasionó el cuadro clínico de impacción gástrica unas seis horas después.

\section{Discusión}

En libertad, los caballos pasan pastando de 12 a 16 horas diarias en periodos de 2 a 3 horas alternados con momentos de descanso e interacción social (Ralston, 1984). Así pues, el aparato digestivo de los caballos se ha adaptado para poder ingerir grandes cantidades de hierba de forma muy repartida a lo largo del día (Hussein y Vogedes, 2007), con independencia de su contenido de fibra (Duncan et al., 1990). Sin embargo, se estima que la ingesta voluntaria de alimento por parte de los caballos alojados en boxes en términos de materia seca es entre el 2 a $2.5 \%$ del peso vivo $(\mathrm{PV})$ en yeguas lactantes y potros y de 1.5 a $2 \%$ PV en yeguas gestantes y caballos, con el fin de prevenir la ocurrencia de trastornos digestivos, como el cólico abdominal agudo, debido a la escasa capacidad gástrica que poseen los équidos (Martínez, 2007).
El cólico abdominal agudo es la patología más frecuente en la clínica médica equina (Singer et al., 2002; Abutarbush et al., 2005), siendo la causa más común la «impacción gástrica» debida a la sobrecarga de cantidades anormalmente elevadas de forraje fibroso de muy baja digestibilidad o concentrado altamente fermentable (White, 2005), que ocasionan la distensión de la víscera. La impacción gástrica implica la sobrecarga del órgano, en este caso con alimento concentrado que se comporta como elemento osmótico, sumado a ello la capacidad de secreción de ácido clorhídrico, haciendo que se retenga líquido e hidrogeniones, lo que lleva a deshidratación e hipocloremia (Radostits et al., 2007).

El signo más importante del cólico es el dolor y su evaluación permite categorizar la gravedad del mismo, ya que existe una buena correlación entre la intensidad del dolor y la gravedad del cólico. El dolor se manifiesta con una gran variedad de signos clínicos como son el comportamiento y la actitud que adopta el animal, el pulso y la frecuencia cardiaca, entre otros. No obstante, conviene considerar que no todos los animales tienen la misma sensibilidad dolorosa y que, a veces, cólicos relativamente simples cursan con dolor intenso. Asimismo, no todos los caballos muestran todos los signos y no todos los que tienen la misma patología demuestran un cuadro clínico similar (Velázquez, 1992).

Dentro del examen físico, es importante medir el tiempo de llenado capilar, el cual está en relación directa con la perfusión a nivel capilar o micro perfusión, y que en caballos deshidratados o en shock hay una demora en el tiempo de llenado. En el mismo orden de importancia se encuentran la humedad y color de las mucosas, donde la humedad refleja el estado de hidratación de manera similar al tiempo de llenado capilar y el color puede indicar congestión venosa o cianosis cuando existe un compromiso más severo (Lemus, 1993). Asimismo, en caballos con dolor o deshidratación de moderada a severa, la frecuencia cardiaca se encuen- 
tra elevada debido a que se estimula el sistema autónomo simpático para que el incremento de los latidos cardiacos mejore la perfusión sanguínea (Gonzales, 2014).

En el examen de la cavidad abdominal no se percibieron sonidos a la auscultación, lo cual se encuentra frecuentemente asociado a cambios morfológicos irreversibles (Gonzales, 2014). Otro aspecto importante a considerar fue la temperatura rectal, que en este caso fue de $37^{\circ} \mathrm{C}$, un valor ligeramente más bajo del rango aceptable; lo que resulta valioso conocer, ya que los caballos que entran en shock presentan extremidades frías y pulso débil (Smith, 2002).

El diagnóstico definitivo de la impacción gástrica es difícil. Si el caballo no ha comido durante varias horas, o si se recupera material alimenticio pobremente macerado o digerido de la sonda nasogástrica, se considera indicativo de la existencia de una impacción gástrica. La clave para realizar el diagnóstico es la incapacidad del estómago para vaciarse de forma apreciable en 12 o 24 horas (Smith, 2010). El paso de la sonda nasogástrica y el vaciado del reflujo gástrico son importantes para aliviar la distensión gástrica y prevenir la ruptura (Velázquez et al., 1992). Por otro lado, la impacción gástrica también se puede diagnosticar por laparotomía exploratoria.

\section{Literatura Citada}

1. Abutarbush SM, Calmart JL, Shoemaker $R W .2005$. Causes of gastrointestinal colic in horses in western Canada: 604 cases (1992 to 2002). Can Vet J 46: 800-805.

2. Duncan P, Foose TJ, Gordon IJ, Gakahu CG, Lloyd M. 1990. Comparative nutrient extraction from forages by grazing bovids and equids: a test of the nutritional model of equid/ bovid competition and coexistence. Oecologia 84: 411-418. doi: 10.1007/ BF00329768

3. Ferreira C, Palhares MS, Melo UP, Braga CE, Silva Filho JM, Gheller VA. 2008. Compactações do trato gastrintestinal em 64 equinos: associação entre alimentação, característica das fezes e resolução clínica. Anais VIII Conferência Sul-americana de Medicina Veterinária. Rio de Janeiro.

4. Gonzales LA. 2014. Tesina: Uso de fenilefrina como tratamiento médico del síndrome abdominal agudo por atrapamiento nefroesplénico: estudio de caso. Tesina. México: Universidad Autónoma del Estado de México. 89 p.

5. Hussein HS, Vogedes LA. 2007. Forage nutritional value for equine as affected by forage species and cereal grain supplementation. Professional Anim Scientist 19: 388-397. doi: 10.15232/S1080-7446(15)31445-5

6. Lemus SF. 1993. Síndrome abdominal agudo. Cuando tomar la decisión quirúrgica. Memorias XV Congreso Nacional de la AMMVEE. Colima, México.

7. Martínez MA. 2007. Nutrición de caballos de ocio alimentados a pesebre. Prod Anim Gestión 5: 1-29. [Internet]. Disponible en: http://www.uco.es/ zootecniaygestion/img/pictorex/ 10_10_21_dt12.pdf

8. Radostits OM, Gay CC, Hinchdliff $K W$, Constable PD. 2007. Veterinary Medicine. $10^{\text {th }}$ ed. London: WB Saunders. $2215 \mathrm{p}$.

9. Ralston SL. 1984. Controls of feeding in horses. J Anim Sci 59: 1354-1361. doi: 10.2527/jas1984.5951354x

10. Singer ER, Smith MA. 2002. Examination of the horse with colic: is it medical or surgical? Equine Vet Educ14: 87-96. doi: 10.1111/j.2042-3292.2002.tb00147.x

11. Smith BP. 2010. Medicina interna de grandes animales. $4^{a}$ ed. España: Elsevier. 1868 p. 
12. Velázquez R, McClure J, Masri D. 1992. Reporte de celiotomías exploratorias en caballos con dolor abdominal agudo. Memorias XIV Congreso Nacional de la AMMVEE. Aguascalientes, México.
13. White NA. 2005. Prevalence, demographics and risk factors for colic. Proc American Association of Equine Practitioners Focus Meeting. Québec, Canada. 